\title{
Reconocimiento de caras en la esquizotipia
}

\author{
Leticia García-Álvarez ${ }^{1,2,3 *}$, Serafín Lemos-Giráldez ${ }^{1,3}$, Mercedes Paíno ${ }^{1,3}$, \\ Eduardo Fonseca-Pedrero ${ }^{1,3}$, Nuria Ordóñez-Camblor ${ }^{1}$ y Patricia Burón-Fernández ${ }^{2,3}$ \\ 1 Departamento de Psicología. Universidad de Oviedo \\ 2 Área de Psiquiatría. Universidad de Oviedo \\ 3 Centro de Investigación Biomédica en Red de Salud Mental (CIBERSAM-Oviedo, G05)
}

\begin{abstract}
Resumen: Estudios previos sugieren la existencia de fallos en la percepción de uno mismo en la esquizofrenia y la esquizotipia y, en particular, una percepción inusual de la propia cara y de la de otros en la esquizotipia. El propósito de este estudio fue explorar las posibles diferencias en el reconocimiento de la propia cara y la de otros, entre las personas que puntúan alto y bajo en esquizotipia, además de confirmar si las dimensiones Cognitivoperceptiva y de Desorganización de la esquizotipia, pero no la dimensión Interpersonal, guardan relación significativa con esas anomalías, según lo observado en estudios previos. La muestra estuvo formada por 219 adolescentes y jóvenes no clínicos, (53\% mujeres). Los instrumentos empleados fueron el Self-Face Recognition Questionnaire (SFRQ), el Cuestionario de Personalidad Esquizotípica versión breve (SPQ-B) y el ESQUIZO-Q. La media de edad de los participantes fue de 16.3 años $(D T=1.18)$. Las comparaciones entre quienes puntúan alto y bajo en esquizotipia ofrece apoyo a la hipótesis de que los rasgos positivos (Cognitivo-perceptivos y de Desorganización) correlacionan significativamente con las dificultades en el reconocimiento de caras. Las mujeres mostraron mayores dificultades que los varones en el reconocimiento de caras, probablemente debido la tendencia a puntuar más alto en la dimensión Cognitivo-perceptiva.
\end{abstract}

Palabras clave: Reconocimiento de caras; esquizotipia; adolescencia; trastornos del yo.

\section{Introducción}

La noción del yo (o self, en inglés) no es un mero constructo teórico o ficción sino que representa una realidad mental en sí misma, siendo interesante conocer los mecanismos que nos permiten reconocernos a nosotros mismos y distinguirnos de los de los demás, ya que es la forma en la que nos construimos como entidades independientes del resto del mundo. Además, es un prerrequisito para atribuir un comportamiento a uno mismo o a los demás y nos permite comunicarnos con otras personas. El auto-reconocimiento depende en gran medida de la capacidad de reconocer nuestras propias acciones. La habilidad para atribuir una acción a su agente parece ser la clave del auto-reconocimiento (Jeannerod, 2003), y el reconocimiento de uno mismo podría ser un indicador de la auto-conciencia o insight. De manera específica, aquellos individuos que reconocen su propia cara son capaces de realizar otras tareas de auto-conocimiento, como la introspección, la cual marcaría las diferencias entre humanos y otras especies (Focquaert, Braeckman, \& Platek, 2008).

El reconocimiento del propio cuerpo no siempre es fácil, particularmente de la propia cara (Kircher et al., 2001). Por ejemplo, cuando una persona se ve a sí misma en un espejo,

* Dirección para correspondencia [Correspondence address]: Leticia García Álvarez. Centro de Investigación Biomédica en Red de Salud Mental, CIBERSAM. Área de Psiquiatría - Universidad de Oviedo. C/ Julián Clavería, 6. 33006. Oviedo (España). E-mail: lettti@gmail.com
Title: Face recognition in schizotypy.

Abstract: Studies suggest disturbances of self in schizophrenia and in schizotypy, and unusual perception of own and other faces in schizotypy. The purpose of this study was to explore possible differences between high and low scores on schizotypal questionnaires and self and others-face recognition, and confirm whether the cognitive-perceptual and disorganization dimensions of schizotypy, but not the interpersonal dimension, significantly correlated with such disturbances, as observed in previous studies. A sample of 219 nonclinical adolescent and young subjects $(53 \% \mathrm{fe}$ males) completed the Self-Face Recognition Questionnaire (SFRQ), the Schizotypal Personality Questionnaire-Brief (SPQ-B), and the Oviedo Schizotypy Assessment Questionnaire (ESQUIZO-Q). Mean age of the participants was 16.3 years $(S D=1.18)$. Comparisons between those participants scoring high and low in schizotypal traits offer further support that positive (Cognitive-perceptual and Disorganization) traits significantly correlate with face recognition difficulties; demonstrating girls more disturbances than boys in face recognition, probably due to higher scores in the Cognitive-perceptive dimension.

Key words: Face recognition; schizotypy; adolescence; self disorders.

existe una discontinuidad entre la parte que está viendo y el resto del cuerpo; siendo necesario un proceso activo para integrar esa parte en una representación total del cuerpo: el esquema corporal (Gallagher, 1995, 2000).

La denominada sintomatología positiva (alucinaciones, delirios), que está presente en las psicosis, podría representar una alteración de los mecanismos que normalmente delimitan los límites entre uno mismo y los demás, o anomalías en la auto-percepción (Sass, 2003). Se ha comprobado que estos pacientes atribuyen sus propias acciones o pensamientos a una fuente externa (Frith, 2004), muestran dificultad para diferenciar su propia voz de la de otras personas y, en consecuencia, tienden a malinterpretar su voz, como si fuese producida por un extraño (Allen et al., 2004), y tienen alterada la percepción del propio cuerpo.

Los trastornos a nivel sensorial (despersonalización y desrealización) suelen guardar relación con la representación simbólica de los estímulos percibidos y se expresan como aberraciones perceptivas de uno mismo, del cuerpo y del mundo (Stanghellini, 2000).

Los trastornos del yo en la esquizofrenia han sido siempre reconocidos como componentes esenciales de su caracterización clínica, y diversos estudios han mostrado que algunos de esos fallos se manifiestan mucho antes de la aparición del trastorno (Moller \& Husby, 2000; Parnas, 2003; Parnas \& Handest, 2003), pudiendo agravarse significativamente durante el curso de la enfermedad (Parnas, Handest, Saebye, \& Hansson, 2003). La entrevista semiestructurada EASE (Examination of Anomalous Self-Experience) fue diseñada, 
precisamente, para el examen cualitativo de los trastornos de la auto-conciencia (Parnas et al., 2005).

Diversos estudios han demostrado también que sutiles alteraciones en la percepción del yo se observan en personas con marcada esquizotipia. De manera específica, Platek y colaboradores han comprobado que las personas que puntúan alto en escalas de esquizotipia presentan tiempos de reacción más lentos que las personas normales en tareas de identificación de imágenes que representan la propia cara o caras de extraños (Platek et al., 2005; Platek et al., 2006). En general, la existencia de importantes déficits en la cognición social y, en particular, dificultades en el reconocimiento de caras en personas de alto riesgo de psicosis y en individuos con rasgos esquizotípicos ha quedado demostrada en recientes investigaciones (Dickey et al., 2012; Fusar-Poli et al., 2012; Thompson et al., 2012; van Rijn, Schothorst, \& van 't Wout, 2011).

Es posible que exista un sistema neuronal especializado en el procesamiento de la propia cara, ya que la gente normal percibe mejor propia cara que la cara de otras personas y el tiempo de reacción en su identificación es menor (Tong \& Nakayama, 1999). Supuestamente, el hemisferio derecho está implicado en el procesamiento de la información sobre uno mismo, y existiría alguna alteración en personas con alta esquizotipia en el procesamiento llevado a cabo por dicho hemisferio o en la transmisión inter-hemisférica (Platek, et al., 2005; Platek, et al., 2006; Sugiura et al., 2005). En cualquier caso, y a modo de especulación, también puede tener sentido aquí aludir al punto de vista de Goldberg (2002), quien refiere que, si bien cada hemisferio cerebral está implicado en todos los procesos cognitivos, su grado de implicación relativa varía de acuerdo con el principio de novedadrutina. El papel especial desempeñado por los lóbulos frontales y el hemisferio derecho, en tratar con la novedad y por el hemisferio izquierdo en el procesamiento de estímulos familiares o la implementación de tareas rutinarias, sugiere la existencia de cambios dinámicos asociados con el aprendizaje, que provocan que el lugar del control cognitivo se desplace desde el hemisferio derecho al hemisferio izquierdo, y desde las partes frontales a las partes posteriores de la corteza. La citada dificultad observada en la auto-percepción, en la esquizofrenia y en la esquizotipia, tal vez pudiera reflejar alguna anomalía en los normales cambios dinámicos derivados del aprendizaje.

La constatación de anomalías en la auto-percepción en la esquizofrenia y en la personalidad esquizotípica indica, una vez más, la existencia de un continuo en la fenomenología y en la etiología de todo el espectro esquizofrénico; si bien, las experiencias anómalas de la esquizotipia no alcanza la intensidad de la psicosis, por cuanto en aquélla la persona es capaz de mantener cierta distancia con su experiencia alterada, una distancia frecuentemente expresada por medio de la expresión condicional "como si"; p. e., "Siento como si mi cuerpo no me perteneciese". La transición a la psicosis se produce cuando estas experiencias subjetivas anómalas lle- gan a ser convicciones, convirtiéndose en delirios, alucinaciones o experiencias de pasividad.

En un reciente estudio realizado por Larøi, D’Argembeau, Brédart y van der Linden (2007) sobre los fallos en el reconocimiento de caras en una muestra no clínica de 170 personas de entre 18 y 57 años, basado en otro anterior de Brédart y Young (2004), se ha desarrollado un breve instrumento para investigar las relaciones existentes entre las tres principales dimensiones (cognitivo-perceptiva, interpersonal y de desorganización) de la esquizotipia, y las alteraciones en el reconocimiento de caras. Dicho instrumento (Self-Face Recognition Questionnaire; SFRQ) ha demostrado tener una estructura trifactorial formada por: a) dificultades en el reconocimiento de la propia cara; b) percepción inusual de la propia cara o de otras caras; y c) dificultades en el reconocimiento de la cara de otras personas. Larøi et al. (2007) encontraron una correlación significativa entre las dimensiones Cognitivo-perceptiva y de Desorganización de la esquizotipia (es decir, las dimensiones más relacionadas con los rasgos o síntomas positivos y cognitivos) y los factores del SFRQ. Sin embargo la dimensión Interpersonal (los rasgos o síntomas negativos) de la esquizotipia no mostró correlación estadísticamente significativa con las medidas del cuestionario.

El propósito del presente estudio fue corroborar, en población adolescente y juvenil española, la posible relación creciente entre esquizotipia psicométrica y dificultad en el reconocimiento de la propia cara o la de otras personas, así como someter a prueba la supuesta mayor dificultad en dicho reconocimiento en individuos que puntúan alto en las dimensiones Cognitivo-perceptiva y de Desorganización de la esquizotipia, pero no en la dimensión Interpersonal. Los análisis correlacionales consideran la esquizotipia como rasgo dimensional, mientras que en las comparaciones de grupos extremos se considera la esquizotipia como fenómeno categorial (Claridge, 1997).

\section{Método}

\section{Participantes}

La muestra estudiada se formó con 219 adolescentes y jóvenes de dos institutos de educación secundaria del Principado de Asturias, de los cuales 115 (53\%) fueron mujeres. La edad media de los participantes fue de $16.3(D T=1.18)$, con un rango de edad de 14 a 19 años. Los institutos pertenecían al sistema educativo público y eran de un nivel socioeconómico medio.

\section{Instrumentos}

Cuestionario Oviedo para la Evaluación de la Esquizotipia (ESQUIZO-Q) (Fonseca, Muñiz, Lemos, Paino, \& Villazón, 2010; Fonseca Pedrero, Paino, Lemos Giráldez, Vallina Fernández, \& Muñiz, 2010). El ESQUIZO-Q es un autoinforme desarrollado para la evaluación de rasgos esquizotípi- 
cos en adolescentes, también utilizable con fines epidemiológicos. Se fundamenta en los criterios diagnósticos del DSM-IV-TR (American Psychiatric Association, 2000) y en el modelo de esquizotaxia de Meehl $(1962,1989)$ sobre predisposición genética a la esquizofrenia. Los ítems del ESQUIZO-Q fueron seleccionados a partir de una revisión exhaustiva de la literatura acerca de la esquizotipia y constructos relacionados (Fonseca-Pedrero et al., 2008) y su construcción se realizó según los pasos propuestos para la elaboración de tests (Schmeiser \& Welch, 2006) y las directrices para la formulación de ítems de elección múltiple (Moreno, Martínez, \& Muñiz, 2006; Muñiz \& Bartram, 2007). El formato de respuesta es tipo Likert de 5 categorías siendo 1 "completamente en desacuerdo" y 5 "completamente de acuerdo". El ESQUIZO-Q consta de un total de 10 subescalas derivadas empíricamente mediante técnicas factoriales: Ideación Referencial, Pensamiento Mágico, Experiencias Perceptivas Extrañas, Pensamiento y Lenguaje Extraños, Ideación Paranoide, Anhedonia Física, Anhedonia Social, Comportamiento Extraño, Ausencia de Amigos Íntimos y Ansiedad Social Excesiva. Estas subescalas se agrupan en tres dimensiones generales de segundo orden: Distorsión de la Realidad (Positiva), Negativa y Desorganización Interpersonal. La construcción y validación del ESQUIZO-Q se realizó con una muestra de 1653 adolescentes no clínicos, y presentó adecuadas propiedades psicométricas. Los niveles de consistencia interna para las subescalas oscilaron entre .62 y .90 , y se obtuvieron diversas evidencias de validez con otros autoinformes que valoran sintomatología emocional y comportamental (Fonseca Pedrero et al., 2011; Fonseca Pedrero, et al., 2010).

Cuestionario de Personalidad Esquizotípica-versión breve (SPQB) (Schizotypal Personality Questionnaire- Brief Form) (Raine \& Benishay, 1995) es un autoinforme de 22 ítems (con respuesta dicotómica Sí/No) desarrollado para la medición de los rasgos esquizotípicos a partir del SPQ (Raine, 1991) y de acuerdo con los criterios del DSM-III-R (American Psychiatric Association, 1987). Proporciona información sobre las tres dimensiones de la esquizotipia: Cognitivoperceptiva, Desorganización Cognitiva, e Interpersonal. Sus propiedades psicométricas se han estudiado en pacientes con esquizofrenia y familiares de primer grado de pacientes con trastornos del espectro esquizofrénico (Bora \& Veznedaroglu, 2007; Compton, Goulding, Bakeman, \& McClure-Tone, 2009), en pacientes adolescentes ambulatorios (Axelrod, Grilo, Sanislow, \& McGlashan, 2001), y ha sido traducido a diferentes idiomas. El SPQ-B es uno de los instrumentos más ampliamente aceptados en la literatura internacional, con adecuadas propiedades psicométricas referidas tanto a la consistencia interna como a sus diferentes evidencias de validez. La versión española utilizada en este estudio (Fonseca-Pedrero, Paíno-Piñeiro, Lemos-Giráldez, Villazón-García, \& Muñiz, 2009) fue validada sobre una muestra de 1683 adolescentes, ofreciendo índices de consistencia interna de las subescalas que oscilaron entre .61 a .81. Los análisis factoriales confirmatorios indicaron que la es- tructura de la prueba se ajustó razonablemente bien tanto al modelo de tres factores (Positivo, Negativo y de Desorganización) como al de cuatro factores (Positivo, paranoide, Negativo y de Desorganización).

Self-Face Recognition Questionnaire (SFRQ) (Larøi, et al., 2007). Evalúa dificultades en el reconocimiento de caras y está formado por 6 ítems, tres de los cuales se refieren a fallos, anomalías o extrañeza, descritas por Brédart y Young (2004), en el reconocimiento de la propia cara (p. e., "Cuando me observo a mí mismo en un espejo, un escaparate, un vídeo o una foto, a veces no me he reconocido, aun cuando no confundí mi cara con la cara de otra persona"), y otros tres en el reconocimiento de la cara de otras personas (p. e., "Alguna vez la cara de alguien que conozco me pareció extraña o rara, aun cuando era consciente de que era su cara”). Cuando la respuesta a un ítem es "Sî", se formulan preguntas complementarias (en un formato tipo Likert de 4 puntos) que exploran: la frecuencia con que ocurre la experiencia; el grado de malestar que genera, y el grado de cansancio/distracción cuando dichos fallos tuvieron lugar. Se ha realizado la adaptación española de la prueba para esta investigación. Como se ha señalado más arriba, los autores obtuvieron una estructura trifactorial.

\section{Procedimiento}

La administración de los cuestionarios se llevó a cabo de forma colectiva, en grupos de 4 a 28 estudiantes, en un espacio temporal específico para un estudio más amplio sobre la detección del riesgo de trastornos psicóticos. Se informó en todo momento de la confidencialidad de las respuestas, así como del carácter voluntario de la participación. No se ofreció gratificación alguna por la participación en el estudio. La investigación fue aprobada por el comité ético de la Universidad de Oviedo.

\section{Análisis de datos}

El análisis de los datos se dividió en tres apartados: 1) para observar si, en el en el reconocimiento de caras, existen diferencias intersexos y entre las personas que puntúan alto y bajo en esquizotipia se realizaron pruebas $t$ de Student para muestras independientes; 2) para comprobar la relación entre el reconocimiento de caras y las dimensiones de esquizotipia se utilizaron las mismas pruebas estadísticas; y 3) analizadas las propiedades métricas del SFRQ, se examinó la estructura interna mediante análisis factorial exploratorio (AF) de componentes principales.

\section{Resultados}

Los valores obtenidos en cada una de las variables utilizadas en el estudio, se presentan en la Tabla 1.

Tabla 1. Análisis descriptivo de las variables. 


\begin{tabular}{lll}
\hline Variables & Media (DT) & Rango \\
\hline Reconocimiento de caras (SFRQ) & $1.81(1.29)$ & $0-5$ \\
\hline Esquizotipia (ESQUIZO-Q) & $95.63(18.15)$ & $57-153$ \\
Factor Distorsión de la realidad & $32.80(9.47)$ & $21-64$ \\
Factor Anhedonia & $14.96(3.88)$ & $9-27$ \\
Factor Desorganización interpersonal & $47.85(11.14)$ & $26-87$ \\
\hline Esquizotipia (SPQ-B) & $6.39(4.07)$ & $0-20$ \\
Factor Cognitivo-perceptivo & $1.65(1.67)$ & $0-7$ \\
Factor Interpersonal & $3.18(2.10)$ & $0-8$ \\
Factor de Desorganización & $1.55(1.45)$ & $0-6$ \\
\hline
\end{tabular}

Análisis de diferencias entre varones y mujeres en el reconocimiento de caras y en la puntuación total de las escalas de esquizotipia

Los resultados muestran diferencias estadísticamente significativas entre ambos sexos; siendo las mujeres quienes presentan mayor dificultad $(t=-2.64 ; p=.009)$. Por el contrario, no se encontraron diferencias estadísticamente significativas entre varones y mujeres en las puntuaciones de esquizotipia.

Para comprobar la posible existencia de diferencias significativas en función de la edad, se formaron tres grupos (1415 años, 16-17 años y 18-19 años), con respecto a las variables previamente comentadas, se llevó a cabo un análisis multivariado de varianza (MANOVA), que no reveló diferencias cuando se consideró todos los datos en conjunto $(\lambda$ de Wilks $=.022 ; p=.462)$. Los datos sobre la frecuencia, el grado de malestar y el nivel de cansancio/distracción de los participantes cuando apreciaron dificultad en el reconocimiento de la propia cara o la de otros, indicaron que les ocurró entre "en muy raras ocasiones" y "en raras ocasio- nes"; en cuyo caso, atribuyen este tipo de fallos más a cansancio/distracción que a estrés.

\section{Relaciones entre el reconocimiento de caras y la es- quizotipia}

Teniendo en cuenta que el primer propósito de esta investigación era observar si existen diferencias en el reconocimiento de caras entre quienes muestran alta y baja esquizotipia, para maximizar las diferencias se seleccionaron a los sujetos extremos, que puntuaron por debajo del percentil 20 en esquizotipia (puntuación menor de 2 en el SPQ-B y menor de 80 en el ESQUIZO-Q ) y los que estaban por encima del percentil 80 (puntuación mayor de 10 en el SPQ-B y mayor de 109 en el ESQUIZO-Q). La comparación ofreció diferencias en el reconocimiento de caras entre ambos grupos, presentando mayor dificultad quienes muestran alta esquizotipia.

Al comparar ambos grupos, formados a partir de las puntuaciones del SPQ-B, se encontraron diferencias estadísticamente significativas en los tres primeros ítems del cuestionario de reconocimiento de caras (SFRQ), pero no en los 3 siguientes; lo cual indica que a mayor puntuación en esquizotipia, más dificultad o extrañeza en el reconocimiento de su propia cara, pero no en el reconocimiento de la cara de otras personas. En cambio, la comparación de los grupos derivados del ESQUIZO-Q ofrece también diferencias estadísticamente significativas en dos ítems relativos al reconocimiento de la propia cara $(2$ y 3$)$ y en un ítem relativo al reconocimiento de la cara de otras personas (6) (Tabla 2).

Tabla 2. Resultados de la comparación intergrupo (alto/bajo esquizotipia en SPQ-B y ESQUIZO-Q) en el reconocimiento de caras.

\begin{tabular}{|c|c|c|c|c|}
\hline & Alta esquizotipia $(n=45)$ & Baja esquizotipia $(n=36)$ & & \\
\hline Variables SFRQ y SPQ-B & $\operatorname{Media}(D T)$ & Media $(D T)$ & $t$ & $p$ \\
\hline Reconocimiento de caras: & $12.59(2.33)$ & $1.42(.79)$ & -3.48 & .001 \\
\hline 1. Mala interpretación self & $0.17(0.38)$ & $0.00(0.00)$ & -2.80 & .006 \\
\hline 2. Fallo reconocimiento self & $0.28(0.46)$ & $0.11(0.31)$ & -2.04 & .045 \\
\hline 3. Self inusual & $0.41(0.50)$ & $0.16(0.37)$ & -2.62 & .011 \\
\hline 4. Otros inusual & $0.46(0.50)$ & $0.29(0.46)$ & -1.59 & .116 \\
\hline 5. Mala interpretación otros & $0.74(0.44)$ & $0.68(0.47)$ & -0.55 & .587 \\
\hline 6. Fallo reconocimiento otros & $0.41(0.50)$ & $0.26(0.45)$ & -1.45 & .150 \\
\hline & Alta esquizotipia $(n=44)$ & Baja esquizotipia $(n=42)$ & & \\
\hline Variables SFRQ y ESQUIZO-Q & Media $(D T)$ & Media $(D T)$ & $t$ & $p$ \\
\hline Reconocimiento de caras: & $1.19(1.70)$ & $0.25(.70)$ & -4.76 & .000 \\
\hline 1. Mala interpretación self & $0.11(0.24)$ & $0.07(0.19)$ & -1.70 & .093 \\
\hline 2. Fallo reconocimiento self & $0.32(0.47)$ & $0.08(0.16)$ & -4.10 & .000 \\
\hline 3. Self inusual & $0.29(0.47)$ & $0.09(0.11)$ & -3.18 & .002 \\
\hline 4. Otros inusual & $0.19(0.40)$ & $0.10(0.01)$ & -1.88 & .064 \\
\hline 5. Mala interpretación otros & $0.09(0.30)$ & $0.11(0.12)$ & -0.83 & .409 \\
\hline 6. Fallo reconocimiento otros & $0.20(0.39)$ & $0.10(0.00)$ & -2.02 & .046 \\
\hline
\end{tabular}

Relaciones entre el reconocimiento de caras y dimensiones de la esquizotipia

También se valoró si existen diferencias significativas en el reconocimiento de caras entre los participantes que pun- túan alto/bajo en el factor Cognitivo-perceptivo. Para ello, se seleccionaron igualmente los sujetos extremos, situados por debajo del percentil 20 en el factor cognitivo-perceptivo de la esquizotipia (puntuación 0 en el factor cognitivoperceptivo del SPQ-B y menor de 24 en el ESQUIZO-Q) y 
por encima del 80 (puntuación mayor de 3 en el factor cognitivo-perceptivo del SPQ-B y mayor de 40 en el ESQUIZO-Q).

Las comparaciones también revelaron diferencias significativas entre quienes puntuaron alto y bajo en el factor Cognitivo-perceptivo de la esquizotipia (SPQ-B), en todos los ítems del cuestionario que mide reconocimiento de caras, excepto en el 5; siendo quienes obtuvieron mayor puntuación en el factor Cognitivo-perceptivo de la esquizotipia los que presentan mayor dificultad en el reconocimiento tanto de su propia cara como de la cara de otras personas, excepto en lo que se refiere a "confundir la cara de una persona que conoce con la de otra persona". Las diferencias fueron también estadísticamente significativas entre quienes puntuaron alto y bajo en el factor Distorsión perceptiva del ESQUIZO-Q, en todos los ítems del cuestionario que mide reconocimiento de caras, excepto en el 1 (mala interpretación de uno mismo) (Tabla 3).

Tabla 3. Resultados de la comparación intergrupo alto/bajo en factor Cognitivo-perceptivo del SPQ-B y en factor 1: Distorsión de la realidad del ESQUIZO-Q.

\begin{tabular}{|c|c|c|c|c|}
\hline Variables SFRQ y $S P Q-B$ & $\begin{array}{c}\text { Alto factor Cognitivo-perceptivo } \\
(n=53) \\
\text { Media (DT) }\end{array}$ & $\begin{array}{c}\text { Bajo factor Cognitivo-peceptivo } \\
(n=62) \\
\text { Media (DT) }\end{array}$ & $t$ & $p$ \\
\hline Reconocimiento de caras: & $2.64(1.40)$ & $1.06(.87)$ & -7.37 & .000 \\
\hline 1. Mala interpretación self & $0.15(0.36)$ & $0.02(0.13)$ & -2.80 & .006 \\
\hline 2. Fallo reconocimiento self & $0.34(0.48)$ & $0.02(0.13)$ & -5.22 & .000 \\
\hline 3. Self inusual & $0.47(0.50)$ & $0.05(0.21)$ & -6.12 & .000 \\
\hline 4. Otros inusual & $0.50(0.50)$ & $0.13(0.33)$ & -4.93 & .000 \\
\hline 5. Mala interpretación otros & $0.75(0.43)$ & $0.64(0.48)$ & -1.34 & .182 \\
\hline 6. Fallo reconocimiento otros & $0.41(0.50)$ & $0.22(0.42)$ & -2.32 & .022 \\
\hline Variables SFRQ y ESQUIZO-Q & $\begin{array}{c}\text { Alto factor Distorsión realidad } \\
(n=46) \\
\text { Media (DT) }\end{array}$ & $\begin{array}{c}\text { Bajo factor Distorsión realidad } \\
(n=48) \\
\text { Media (DT) }\end{array}$ & $t$ & $p$ \\
\hline Reconocimiento de caras: & $-1.59(2.03)$ & $.22(1.15)$ & -7.15 & .000 \\
\hline 1. Mala interpretación self & $-.05(.15)$ & $.05(.06)$ & -.89 & .375 \\
\hline 2. Fallo reconocimiento self & $-.24(.36)$ & $.06(.12)$ & -3.84 & .000 \\
\hline 3. Self inusual & $-.44(.60)$ & $.08(.28)$ & -5.38 & .000 \\
\hline 4. Otros inusual & $-.38(.57)$ & $.09(.19)$ & -4.03 & .000 \\
\hline 5. Mala interpretación otros & $-.22(.41)$ & $.10(.03)$ & -2.31 & .023 \\
\hline 6. Fallo reconocimiento otros & $-.27(.45)$ & $.09(.09)$ & -2.94 & .004 \\
\hline
\end{tabular}

Se realizaron los mismos análisis, pero tomando la media como criterio para establecer dos grupos (puntuación mayor o menor que 6 en el factor Cognitivo-perceptivo del SPQB), encontrándose los mismos resultados; es decir, las personas con mayor puntuación en esquizotipia presentan más dificultad en el reconocimiento tanto de su propia cara como de la cara de otras personas, excepto en lo que se refiere a "confundir la cara de una persona que conoce con la de otra persona".

Con respecto al factor Interpersonal del SPQ-B y Anhedonia del ESQUIZO-Q, no se observaron diferencias estadísticamente significativas en el reconocimiento de caras entre los que puntúan alto/bajo en dicho factor; sin embargo, sí se encontraron diferencias estadísticamente significativas en el reconocimiento de caras entre los que puntúan alto/bajo en el factor de Desorganización del SPQ-B y el factor Desorganización interpersonal del ESQUIZO-Q, siendo mayor la distorsión perceptiva de uno mismo y de los demás en quienes puntuaron alto en dichas dimensiones.

Se han examinado también las correlaciones entre el reconocimiento de caras y los factores de esquizotipia. Los datos muestran correlaciones significativas entre el reconocimiento de caras y la esquizotipia, medida con el SPQ-B $(r=$ $.314, p=.01)$ y el ESQUIZO-Q $(r=.352, p=.01)$; confirmando que a mayor esquizotipia, más dificultad para el re- conocimiento de caras. La correlación bivariada de toda la muestra entre la dificultad en el reconocimiento de caras y cada uno de los factores de la esquizotipia, medida con el SPQ-B, resulta estadísticamente significativa con el factor Cognitivo-perceptivo $(r=.434, p=.01)$ y con el factor de Desorganización $(r=.218, p=.01)$, pero no con el factor Interpersonal. En el caso del ESQUIZO-Q, la correlación es estadísticamente significativa entre el reconocimiento de caras y el factor Distorsión de la realidad $(r=.441, p=.01)$, y también el factor Desorganización interpersonal $(r=.227, p$ $=.01$ ), pero no con el factor Anhedonia. A mayor intensidad de rasgos o signos cognitivos-perceptivos y de desorganización de la esquizotipia, mayor dificultad en el reconocimiento de caras.

Las correlaciones por sexo entre los factores del SPQ-B y la puntuación total del SFRQ muestra que en los varones sólo hay correlación significativa con el factor Cognitivoperceptivo: a mayor puntuación en el factor Cognitivoperceptivo más anomalías en el reconocimiento de caras (Tabla 4); y en las mujeres todos los factores muestran correlación positiva significativa con la dificultad para reconocer caras.Las correlaciones entre los factores del ESQUIZO-Q y la puntuación total del SFRQ son significativas en el factor Distorsión de la realidad en ambos sexos, y en el factor Desorganización, en los varones. Finalmente, fue correlación 
alta y significativa $(r=.797, p=.01)$ entre la esquizotipia medida con el SPQ-B y medida con el ESQUIZO-Q; lo cual confirma la convergencia de ambos instrumentos en la evaluación de la esquizotipia.

Tabla 4. Matriz de correlaciones bivariadas entre las dimensiones de esquizotipia y el reconocimiento de caras, en mujeres (por encima de la diagonal) y en varones (por debajo de la diagonal).

\begin{tabular}{|c|c|c|c|c|c|}
\hline & $S P Q-B 1$ & $S P Q-B 2$ & $S P Q-B 3$ & SPQ-B Total & SFRQ \\
\hline \multicolumn{6}{|l|}{ SPQ-B: } \\
\hline 1. Factor Cognitivo-perceptivo & ---- & $.417 * *$ & $.384 * *$ & $.750 * *$ & $.540^{* *}$ \\
\hline 2. Factor Interpersonal & $.307 * *$ & ---- & $.474 * *$ & $.849 * *$ & $.293^{* *}$ \\
\hline 3. Factor de Desorganización & $.402 * *$ & $.505^{* *}$ & ---- & $.748^{* *}$ & $.370^{* *}$ \\
\hline SPQ-B Total & $.719 * *$ & $.819 * *$ & $.785^{* *}$ & ---- & $.497 * *$ \\
\hline \multirow[t]{2}{*}{ SFRQ } & $.311 * *$ & -.024 & .138 & .167 & ---- \\
\hline & ESQUIZO-Q1 & ESQUIZO-Q2 & ESQUIZO-Q3 & ESQUIZO-Q Total & SFRQ \\
\hline \multicolumn{6}{|l|}{ ESQUIZO-Q: } \\
\hline 1. Factor Distorsión de la realidad & ---- & .028 & $.622 * *$ & $.732 * *$ & $.318^{* *}$ \\
\hline 2. Factor Anhedonia & -.109 & ---- & .134 & $.226^{*}$ & .012 \\
\hline 3. Factor Desorganización interpersonal & $.265^{* *}$ & .081 & ---- & $.810^{* *}$ & -.102 \\
\hline ESQUIZO-Q Total & $.851 * *$ & $.266 * *$ & $.919 * *$ & ---- & .126 \\
\hline SFRQ & $.517 * *$ & -.051 & $.448^{* *}$ & $.505^{* *}$ & ---- \\
\hline
\end{tabular}

SFRQ

\section{Análisis de la estructura factorial del SFRQ}

Para analizar la estructura interna del SFRQ se realizó un análisis de componentes principales con posterior rotación Varimax. El índice KMO fue .594 siendo la prueba de la esfericidad de Bartlett $p \leq .001$. Para decidir el número de componentes a conservar se examinó el gráfico de sedimentación. Se obtuvieron tres componentes que explicaron un $62.35 \%$ de la varianza total. Estos resultados concuerdan con el Criterio de Kaiser (valores propios mayores que la unidad). Los tres primeros eigenvalues fueron: 1.615 para el primer factor, 1.138 para el segundo y 0.988 para el tercero (en este caso, un valor próximo a 1). En la Tabla 5 se presentan los resultados del estudio del análisis de al estructura interna. Para observar las relaciones entre los factores del cuestionario de reconocimiento de caras, se llevaron a cabo correlaciones bivariadas, y los datos muestran correlación positiva estadísticamente significativa entre los componentes I y II del SFRQ en toda la muestra $(r=.18, p=.01)$. En las correlaciones por sexo, se comprueba que esto es así también para los varones, pero no para las mujeres (Tabla 6).

Tabla 5. Análisis de la estructura interna del SFRQ

\begin{tabular}{llcc}
\hline Reconocimiento de caras (SFRQ) & \multicolumn{2}{c}{ Componentes } \\
\hline Items & I & II & III \\
\hline 1. Mala interpretación self & & & .974 \\
2. Fallo reconocimiento self & .728 & & \\
3. Self inusual & .648 & & \\
4. Otros inusual & .653 & & \\
5. Mala interpretación otros & & .842 & \\
6. Fallo reconocimiento otros & & .736 & \\
Eigenvalues: 1.615 factor I, 1.138 factor II y .988 factor III. Se han eliminado \\
las cargas factoriales inferiores a .30. \\
Varianza total explicada: $62.35 \%$.
\end{tabular}

Varianza total explicada: $62.35 \%$.
Tabla 6. Matriz de correlaciones de Pearson entre los factores del SFRQ en mujeres (por encima de la diagonal) y en varones (por debajo de la diagonal). FACTOR 1 FACTOR 2 FACTOR 3

\begin{tabular}{llll} 
FACTOR 1 (items 2, 3 y 4) & ---- & .138 & -.004 \\
FACTOR 2 (items 5 y 6) & $.224^{*}$ & ----- & .073 \\
FACTOR 3 (item1) & .081 & .099 & ------ \\
\hline$* p<.05$
\end{tabular}

\section{Discusión y conclusiones}

El propósito del estudio fue investigar posibles anomalías autopercibidas en el reconocimiento de la propia cara (apreciada en fotos o reflejada en un espejo) y la de otras personas, en adolescentes y jóvenes con rasgos esquizotípicos.

En el estudio se plantearon varias cuestiones a las que se pretendía dar respuesta. Por un lado, se trataba de observar si existen diferencias en el reconocimiento de caras, propia o ajena, ente los participantes que puntúan alto y bajo en esquizotipia, confirmándose que existen dichas diferencias, y en el sentido observado por Larøi et al. (2007). Se ha hipotetizado que la causa de estas dificultades podría ser un fallo específico en el escaneo visual de caras, considerado un caso particular de un problema en el procesamiento de objetos complejos (Williams, Loughland, Gordon, \& Davidson, 1999); o bien un déficit global en el procesamiento visual (Johnson, Lowery, Kohler, \& Turetsky, 2005).

En este estudio, además, se analizaron por primera vez cada uno de los ítems del SFRQ individualmente, encontrándose diferencias significativas en los tres primeros (que se refieren específicamente al reconocimiento de la cara propia) con respecto a los que puntúan alto/bajo en esquizotipia en el SPQ-B; siendo quienes puntúan alto en esquizotipia (SPQ-B) los que más frecuentemente aducen dificultades para reconocer su propia imagen facial. Como se ha señalado, Platek y Gallup (2002) empíricamente comprobaron también que el tiempo de reacción para las imágenes de la propia cara era mayor en las personas que 
obtienen puntuaciones más altas en el SPQ, respecto a quienes obtuvieron puntuaciones bajas. Aunque estos datos no se reprodujeron simétricamente con el ESQUIZO-Q, se confirmó también más dificultad para reconocer la cara propia cuanto más intensa es la esquizotipia. Además, los que puntúan alto en el factor Cognitivo-perceptivo de la esquizotipia mostraron dificultades, no sólo para reconocerse a ellos mismos sino también para reconocer a los demás, respecto a quienes puntúan bajo.

Entre los que puntuaron alto/bajo en el factor Interpersonal del SPQ-B y Anhedonia del ESQUIZO-Q, no se encontraron diferencias estadísticamente significativas en la dificultad en el reconocimiento de caras; pero sí fueron estadísticamente significativas las diferencias en el factor de Desorganizacion del SPQ-B y el factor de Desorganización interpersonal del ESQUIZO-Q. En consecuencia, se confirmó la correlación significativa encontrada por Larøi et al. (2007) entre los factores Cognitivo-perceptivo y de Desorganización y la frecuencia de anomalías en el reconocimiento de caras; si bien con algunas diferencias por sexo. Otro dato que se confirmó fue la ausencia de correlación significativa entre la dimensión Interpersonal de la esquizotipia y los fallos en el reconocimiento de caras; lo cual apoya la hipótesis de que los factores positivo (Cognitivo-perceptivo) y negativo (Interpersonal) de la esquizotipia representan dimensiones neuroconductuales discretas. Los síntomas negativos de la esquizotipia están relacionados con disfunción ejecutiva frontal, con el aumento de la ansiedad social, con el fenómeno obsesivo compulsivo (Dinn, Harris, Aycicegi, Greene, \& Andover, 2002) y con déficit en la expresión emocional (alexitimia, identificación de emociones).

El hecho de que las dimensiones Cognitivo-perceptiva y de Desorganización de la esquizotipia correlacionen significativamente con fallos el reconocimiento de caras confirman también el carácter continuo de este fenómeno, siendo más acusados los fallos en el reconocimiento del self que ocurren en la esquizofrenia (Parnas \& Handest, 2003) que en la esquizotipia (Platek, Myers, Critton, \& Gallup, 2003). Si bien la relación entre el reconocimiento de la propia cara y de la cara de otras personas y los factores Cognitivo-perceptivo y de Desorganización fue estudiada por primera ver por Larøi y colaboradores, a nivel teórico, Sass y Parnas (2003) ya habían sugerido que los síntomas Cognitivo-perceptivos y de Desorganización están asociados con anormalidades en la experiencia del self.

Los resultados, en función del sexo, indicaron que las mujeres presentan mayor dificultad en el reconocimiento de caras; lo cual tiene sentido, por su específica relación con el factor Cognitivo-perceptivo de la esquizotipia y pensamiento divergente, en el cual las mujeres tienden a puntuar más alto (Clapham \& King, 2010).

Otro de los propósitos del estudio fue verificar la estructura trifactorial del SFRQ encontrada por Larøi et al. (2007) en una muestra de edad de 18 a 57 años: 1) dificultades en el reconocimiento de la propia cara (ítems 1 y 2); 2) Percepción inusual de la propia cara o de otras (ítems 3 y 4); y 3) Difi- cultades en el reconocimiento de la cara de otras personas (items 5 y 6).

Con población adolescente y juvenil, la varianza explicada por tres factores es muy similar a la observada por Larøi et al., si bien los factores encontrados en nuestro estudio están formados por los ítems 2, 3 y 4 (factor 1), ítems 5 y 6 (factor 2) ítem 1 (factor 3). Por tanto, los datos coinciden básicamente con los encontrados por Larøi y colaboradores en todos los ítems, excepto en el ítem 2 (que hace referencia a fallos en el reconocimiento de uno mismo). Los análisis correlacionales de Larøi et al. mostraron que los factores 1 (dificultades en el reconocimiento de la cara propia) y 3 (dificultades en el reconocimiento de la cara de otras personas) guardaban correlación significativa. En este estudio, se observó correlación estadísticamente significativa entre el factor 1 y 2 del SFRQ. Quizá estas diferencias sean debidas a las características de la muestra; por lo que sería necesario realizar más estudios para observar si se confirman estos datos.

La asociación entre las dificultades en el reconocimiento y el factor Cognitivo-perceptivo de la esquizotipia requiere mayor análisis. El primer paso para reconocer una cara y juzgar el parecido es obtener información contextual; actividad cognitiva en la cual las personas con esquizofrenia y esquizotipia tienen serías dificultades (Hemsley, 2005; Lemos Giráldez, Vallina Fernández, \& Fernández Iglesias, 2006; Waters, Badcock, Maybery, \& Michie, 2006; Waters, Maybery, Badcock, \& Michie, 2004). El segundo paso, proceso de decisión, puede verse alterado por su tendencia a tomar decisiones rápidamente y basadas en poca información (Moritz \& Woodward, 2006).

Tal y como plantean Larøi et al. (2007), futuras investigaciones deberían examinar la contribución del proceso de decisión y de la obtención de información contextual en el reconocimiento de caras; y también identificar otros procesos cognitivos implicados en los fallos encontrados en personas esquizotípicas, como la inhibición. La asociación entre las dificultades en el reconocimiento y el factor de Desorganización es más difícil de interpretar. Parnas (2003) sugiere que la hiper-reflexividad (exagerada toma de conciencia de uno mismo) y la hipo-reflexividad se deben a anormalidades en el pensamiento, habla y atención, y son, al menos en parte, responsables de las relaciones encontradas entre la Desorganización y las dificultades en el reconocimiento. Otra razón podría ser cierto solapamiento entre Desorganización cognitiva y la esquizotipia positiva (Cognitivo-perceptiva) (Bentall, Claridge, \& Slade, 1989; Mason \& Claridge, 2006; Raine et al., 1994). De todas formas, la relación entre este factor y el reconocimiento de caras no se observó en los varones; por lo que habría que tomar estos datos con cierta precaución.

La población esquizotípica, no clínica, permite estudiar fenómenos presentes en la esquizofrenia, sin que se produzca un efecto de confundido con otro tipo de variables, como medicación, duración de la enfermedad y gravedad de los síntomas, estigma o institucionalización; y los datos encon- 
trados revelan que las anormalidades en el self están presentes también en la población normal, reflejando un rasgo más en los trastornos del espectro esquizofrénico, en un continuo psicopatológico (Fonseca-Pedrero et al., 2010). Como indican Poreh, Whitman, Weber y Ross (1994) los resultados similares en personas con esquizofrenia y en estudiantes con rasgos esquizotípicos sugieren que ambos grupos tienen fallos no sólo en el reconocimiento de caras, sino también de los afectos que reflejan esas caras; debidos tal vez a un déficit generalizado de atención y vigilancia o específico del reconocimiento de emociones (Dickey, et al., 2012; Fusar-Poli, et al., 2012; Thompson, et al., 2012).

Finalmente, deben reconocerse algunas limitaciones en este estudio. La primera se deriva del propio instrumento de medida SFRQ, al no controlar suficientemente las condicio-

\section{Referencias}

Allen, P. P., Johns, L. C., Fu, C. H., Broome, M. R., Vythelingum, G. N., \& McGuire, P. K. (2004). Misattribution of external speech in patients with hallucinations and delusions. Schizophrenia Research, 69, 277-287.

American Psychiatric Association. (1987). Diagnostic and statistical manual of mental disorders (3rd ed. Revised). Washington, DC: Author.

American Psychiatric Association. (2000). Diagnostic and statistical manual of mental disorders: DSM-IV-Tr (4th ed. text revision). Washington, DC: American Psychiatric Association.

Axelrod, S. R., Grilo, C. M., Sanislow, C., \& McGlashan, T. H. (2001). Schizotypal personality questionnaire-brief: Factor structure and convergent validity in inpatient adolescents. Journal of Personality Disorders, 15.

Bentall, R. P., Claridge, G. S., \& Slade, P. D. (1989). The multidimensional nature of schizotypal traits: A factor analytic study with normal subjects. British Journal of Clinical Psychology, 28, 363-375.

Bora, E., \& Veznedaroglu, B. (2007). Temperament and character dimensions of the relatives of schizophrenia patients and controls: The relationship between schizotypal features and personality. European Psychiatry, 22, 27-31.

Brédart, S., \& Young, A. W. (2004). Self-recognition in everyday life. Cognitive Neuropsycbiatry, 9, 183-197.

Clapham, M. M., \& King, W. R. (2010). Psychometric characteristics of the CREA in an English speaking population. Anales de Psicologia/ Annals of Psychology, 26 206-211.

Claridge, G. (1997). Theoretical background and issues. En G. Claridge (Ed.), Schizotypy: Implications for illness and health (pp. 3-18). Oxford: Oxford University Press.

Compton, M. T., Goulding, S. M., Bakeman, R., \& McClure-Tone, E. B. (2009). An examination of the factorial structure of the Schizotypal Personality Questionnaire-Brief (SPQ-B) among undergraduate students. Schizophrenia Research, 115, 286-289.

Dickey, C. C., Vu, M. T., Voglmaier, M. M., Niznikiewicz, M. A., McCarley, R. W., \& Panych, L. P. (2012). Prosodic abnormalities in schizotypal personality disorder. Schizophrenia Research, 142, 20-30.

Dinn, W. M., Harris, C. L., Aycicegi, A., Greene, P., \& Andover, M. S. (2002). Positive and negative schizotypy in a student sample: Neurocognitive and clinical correlates. Schizophrenia Research, 56, 171185.

Focquaert, F., Braeckman, J., \& Platek, S. M. (2008). An evolutionary cognitive neuroscience perspective on human self-awareness and theory of mind. Philosophical Psychology, 21, 47-68.

Fonseca-Pedrero, E., Lemos-Giráldez, E., Paino, M., Villazón-García, U., Sierra-Baigrie, S., \& Muñiz, J. (2009). Experiencias psicóticas atenuadas en población adolescente / Attenuated psychotic experiences in adolescents. Papeles del Psicólogo, 30, 63-73.

Fonseca-Pedrero, E., Lemos-Giráldez, S., Paino, M., Sierra- Baigrie, S., Villazón-García, U., García-Portilla González, M. P. \& Muñiz, J. (2010). nes y circunstancias en las que esos fallos en el reconocimiento de caras tienen lugar. Además, en el rango de edad de los sujetos evaluados, las escalas de esquizotipia podrían interpretar como anómalas ciertas características típicas de la adolescencia, que forman parte de los propios procesos madurativos del desarrollo (Fonseca-Pedrero et al., 2009).

Futuras investigaciones deberían mejorar el SFRQ, de manera que sea más comprensible y que delimite mejor las circunstancias en las que ocurren esos fallos en el reconocimiento.

Agradecimientos.- Los autores expresan su agradecimiento al Ministerio de Ciencia e Innovación (ref. MICINN-08-PSI2008-06220) por el apoyo en la realización de este estudio.

Dimensionality of hallucinatory predisposition: Confirmatory factor analysis of the Launay-Slade Hallucination Scale-revised in college students. Anales de Psicologial Annals of Psychology, 26, 41-48.

Fonseca-Pedrero, E., Paíno-Piñeiro, M., Lemos-Giráldez, S., VillazónGarcía, U., \& Muñiz, J. (2009). Validation of the Schizotypal Personality Questionnaire-Brief Form in adolescents. Schizophrenia Research, 111, 53-60.

Fonseca-Pedrero, E., Paíno, M., Lemos-Giráldez, S., García-Cueto, E., Campillo-Álvarez, A., Villazón-García, U. \& Muñiz, J. (2008). Schizotypy assessment: State of the art and future prospects. International Journal of Clinical and Health Psychology, 8, 577-593.

Fonseca, E., Muñiz, J., Lemos, S., Paino, M., \& Villazón, U. (2010). EsquizoQ: Cuestionario Oviedo para la evaluación de la esquirotipia. Madrid: TEA Ediciones.

Fonseca Pedrero, E., Paino, M., Lemos Giráldez, S., Sierra Baigrie, S., Ordóñez Camblor, N., \& Muñiz, J. (2011). Early psychopathological features in Spanish adolescents. Psicothema, 23, 87-93.

Fonseca Pedrero, E., Paino, M., Lemos Giráldez, S., Vallina Fernández, O., \& Muñiz, J. (2010). ESQUIZO-Q: Un instrumento para la valoración del "alto riesgo psicométrico" a la psicosis / ESQUIZO-Q: An instrument for the assessment of "psychometric high risk" for psychosis. Clínica y Salud, 21, 255-269.

Frith, C. D. (2004). Schizophrenia and theory of mind. Psychological Medicine, 34, 385-389.

Fusar-Poli, P., Deste, G., Smieskova, R., Barlati, S., Yung, A. R., Howes, O., ... Borgwardt, S. (2012). Cognitive functioning in prodromal psychosis: A meta-analysis. Archives of General Psychiatry, 69, 562-571.

Gallagher, S. (1995). Body schema and intentionality. En J. L. Bermudez, A. Marcel \& N. Eilan (Eds.), The body and the self (pp. 225-244). Cambridge, MA: MIT Press.

Gallagher, S. (2000). Philosophical conceptions of the self: implications for cognitive science. Trends in Cognitive Sciences, 4, 14-21.

Goldberg, E. (2002). El cerebro ejecutivo: Lóbulos frontales y mente civilizada. Barcelona: Crítica.

Hemsley, D. R. (2005). The schizophrenic experience: Taken out of context? Schizophrenia Bulletin, 31, 43-53.

Jeannerod, M. (2003). The mechanism of self-recognition in humans. Behavioural Brain Research, 142, 1-15.

Johnson, S. C., Lowery, N., Kohler, C., \& Turetsky, B. I. (2005). Globallocal visual processing in schizophrenia: evidence for an early visual processing deficit. Biological Psychiatry, 58, 937-946.

Kircher, T. T. J., Senior, C., Phillips, M. L., Rabe-Hesketh, S., Benson, P. J., \& Bullmore, E. T. (2001). Recognizing one's own face. Cognition and Emotion, 78, 1-15.

Larøi, F., D’Argembeau, A., Brédart, S., \& van der Linden, M. (2007). Face recognition failures in schizotypy. Cognitive Neuropsychiatry, 12, 554-571. 
Lemos Giráldez, S., Vallina Fernández, O., \& Fernández Iglesias, P. (2006). La investigación cognitiva del síndrome esquizofrénico. Apuntes de Psicologia, 24, 31-49

Mason, O., \& Claridge, G. (2006). The Oxford-Liverpool Inventory of Feelings and Experiences (O-LIFE): Further description and extended norms. Schizophrenia Research, 82, 203-211.

Meehl, P. E. (1962). Schizotaxia, schizotypy, schizophrenia. American Psychologist, 17, 827-838.

Meehl, P. E. (1989). Schizotaxia revisited. Archives of General Psychiatry, 46, 935-944.

Moller, P., \& Husby, R. (2000). The initial prodrome in schizophrenia: Searching for naturalistic core dimensions of experience and behaviour. Schizophrenia Bulletin, 26, 217-232.

Moreno, R., Martínez, R., \& Muñiz, J. (2006). New guidelines for developing multiple-choice items. Methodology, 2, 65-72.

Moritz, S., \& Woodward, T. S. (2006). The contribution of metamemory deficits to schizophrenia. Journal of Abnormal Psychology, 155, 15-25.

Muñiz, J., \& Bartram, D. (2007). Improving international tests and testing. European Psychologist, 12, 206-219.

Parnas, J. (2003). Self and schizophrenia: A phenomenological perspective. En T. Kircher \& A. David (Eds.), The self in neuroscience and psychiatry (pp. 217-241). Cambridge, UK: Cambridge University Press.

Parnas, J., \& Handest, P. (2003). Phenomenology of anomalous selfexperience in early schizophrenia. Comprehensive Psychiatry, 44, 121-134.

Parnas, J., Handest, P., Saebye, D., \& Hansson, L. (2003). Anomalies of subjective experience in schizophrenia and psychotic bipolar illness. Acta Psychiatrica Scandinavica, 108, 126-133.

Parnas, J., Moller, P., Kircher, T. T. J., Thalbitzer, J., Jansson, L., Handest, P. \& Zahavi, D. (2005). EASE: Examination of Anomalous SelfExperience. Psychopathology, 38, 235-258.

Platek, S. M., Fonteyn, L. C. M., Izzetoglu, M., Myers, T. E., Ayaz, H., Li, C. \& Chance, B. (2005). Functional near infrared spectroscopy reveals differences in self-other processing as a function of schizotypal personality traits. Schizophrenia Research, 73, 125-127.

Platek, S. M., \& Gallup, G. G. (2002). Self-face recognition is affected by schizotypal personality traits. Schizophrenia Bulletin, 57, 311-315.

Platek, S. M., Loughead, J., Gur, R. C., Busch, S., Ruparel, K., Phend, N., ... Langleben, D. D. (2006). Neural substrates for functionally discriminating self-face from personality familiar faces. Human Brain Mapping, 27, 91-98.

Platek, S. M., Myers, T. E., Critton, S. R., \& Gallup, G. G. (2003). A lefthand advantage for self-description: The impact of schizotypal personality traits. Schizophrenia Research, 65, 147-151.

Poreh, A. M., Whitman, R. D., Weber, M., \& Ross, T. (1994). Facial recognition in hypothetically schizophrenic college students. Journal of Nervous and Mental Disease, 182, 503-507.
Raine, A. (1991). The SPQ: A scale for the assessment of Schizotypal Personality Disorder based on DSM-III-R criteria. Schizophrenia Bulletin, 17, 555-564.

Raine, A., \& Benishay, D. (1995). The SPQ-B: A brief screening instrument for schizotypal personality disorder. Journal of Personality Disorders, 9, 346355 .

Raine, A., Reynolds, C., Lencz, T., Scerbo, A., Triphon, N., \& Kim, D. (1994). Cognitive-perceptual, interpersonal, and disorganized features of schizotypal personality. Schizophrenia Bulletin, 20, 191-201.

Sass, L. A. (2003). Self-disturbance in schizophrenia: Hyperreflexivity and diminished self-affection. In T. Kircher \& A. David (Eds.), The self in neuroscience and psycbiatry (pp. 242-271). Cambridge, UK: Cambridge University Press.

Sass, L. A., \& Parnas, J. (2003). Schizophrenia, consciousness, and the self. Schizophrenia Bulletin, 29, 427-444.

Schmeiser, C. B., \& Welch, C. (2006). Test development. En R. L. Brennan (Ed.), Educational Measurement (4th ed.) (pp. 307-353). Westport, CT: American Council on Education/Praeger.

Stanghellini, G. (2000). Vulnerability to schizophrenia and lack of common sense. Schizophrenia Bulletin, 26, 775-787.

Sugiura, M., Watanabe, J., Maeda, Y., Matsue, Y., Fukuda, H., \& Kawashima, R. (2005). Cortical mechanisms of visual self-recognition. Neuroimage, 24, 143-149.

Thompson, A., Papas, A., Bartholomeusz, C., Allott, K., Amminger, P., Nelson, B., ... Yung, A. (2012). Social cognition in clinical "at risk" for psychosis and first episode psychosis populations. Schizophrenia Research, 141, 204-209.

Tong, F., \& Nakayama, K. (1999). Robust representations for faces: Evidence from visual search. Journal of Experimental Psychology: Human Perception \& Performance, 25, 1016-1035.

van Rijn, S., Schothorst, P., \& van 't Wout, M. (2011). Affective dysfunctions in adolescents at risk for psychosis: Emotion awareness and social functioning. Psychiatry Research, 187, 100-105.

Waters, F. A. V., Badcock, J. C., Maybery, M. T., \& Michie, P. T. (2006). Auditory hallucinations in schizophrenia: Intrusive thoughts and forgotten memories. Cognitive Neuropsycbiatry, 11, 65-83.

Waters, F. A. V., Maybery, M. T., Badcock, J. C., \& Michie, P. T. (2004). Context memory and binding in schizophrenia. Schizophrenia Research, 68, 119-125.

Williams, L. M., Loughland, C. M., Gordon, E., \& Davidson, D. (1999). Visual scanpaths in schizophrenia: Is there a deficit in face recognition? Schizophrenia Research, 40, 189-199.

(Articulo recibido: 11-4-2011; revisado: 20-12-2012; aceptado: 23-1-2013) 